\title{
The Inverse Marsh Error
}

Frank R. Fronczek, Department of Chemistry, Louisiana State University, Baton Rouge, Louisiana, 70803.

Dick Marsh is probably best known worldwide for his efforts to correct and prevent description of structures in space groups of unnecessarily low symmetry, particularly when an inversion center has been missed. A peril which is less easily detectable is that of using popular space groups when the true symmetry is actually lower, and the structure is pseudosymmetric. Examples will be described in which the structure masquerades as P-1, but is really $\mathrm{P} 1$, as $\mathrm{C} 2 / \mathrm{c}$, but is really $\mathrm{Cc}$, etc. Although a somewhat acceptable refinement is usually possible in the higher-symmetry space group, unnecessary disorder is necessarily present. Incidence of the inverse Marsh error may be increasing for two reasons: Fear of being Marshed when CheckCIF detects pseudosymmetry; the more common use of lowtemperature data, when cooling causes a phase change to a pseudosymmetric polymorph of lower symmetry.

Some highlights of Dick Marsh's career will be presented, along with recollections of crystallography at Caltech in the early1970s. 